\title{
A Bluetooth Audio Amplifier Project for an Embedded Systems Course
}

\section{Dr. James Moscola, York College of Pennsylvania}

James Moscola is an Assistant Professor of Computer Science and Computer Engineering at York College of Pennsylvania. He received a B.S. in Physical Science from Muhlenberg College in 2000, a B.S. in Computer Engineering, a M.S. in Computer Science, and a Ph.D. in Computer Engineering from Washington University in St. Louis in 2001, 2003, and 2008 respectively. His interests include reconfigurable architectures, and embedded systems. 


\title{
A Bluetooth Audio Amplifier Project for an Embedded Systems Course
}

\author{
James Moscola \\ jmoscola@ycp.edu \\ Department of Engineering and Computer Science \\ York College of Pennsylvania
}

\begin{abstract}
This paper describes a design project in which students were required to design and build a Bluetooth audio amplifier for an embedded systems course at York College of Pennsylvania. Requirements for the device include connectivity from audio sources that support the Bluetooth A2DP profile, two-channel stereo amplification at 15 Watts per channel, and a touchscreen display with playback controls and track and artist information. The three main components, the Bluetooth module, the amplifier IC, and the touchscreen LCD, were pre-selected for the students. The remainder of the components, such as analog circuit components, power supplies, voltage regulators, etc., were selected by the students as part of the design process.

This design project is intended to provide students with a complete embedded system design experience. Students start by reading data sheets, selecting components, performing schematic capture of their design, and maintaining a bill of materials. Next, each student creates a PCB layout for their design, generates the required Gerber files, and orders their components and PCBs. When components arrive each student does final assembly, soldering, and debugging of their completed PCBs. The final step is configuring the firmware on the Bluetooth module and developing a user interface on the LCD.
\end{abstract}

\section{Introduction}

The engineering programs at York College of Pennsylvania have always placed a heavy emphasis on hands-on learning. Experiential learning is a core component of these programs from students' very first semester. This paper describes a Bluetooth audio amplifier project that was recently completed by undergraduate computer engineering students in an embedded systems course. The amplifier accepts connections from Bluetooth audio sources such as smartphones, tablets and computers. The wireless audio signal is amplified and output via a standard pair of speakers. Students worked independently and were given 12 weeks to complete the project during their 
final academic semester. At the end of the semester each student had a fully assembled amplifier, of their own design, that they could keep.

The goal of this project was to provide computer engineering students with a complete embedded system design experience. The students started the semester with a list of requirements and a few pre-selected components. They ended the semester assembling, testing, and debugging their designs. Each student was required to maintain and manage their own project and timeline. However, several milestones were provided throughout the semester to assist students in planning. For example, students were required to order their PCBs (printed circuit boards) prior to a certain date to ensure that there was sufficient time remaining in the semester for the PCBs to be manufactured, shipped, assembled, tested, and debugged.

\section{Background}

In recent years, wireless speakers have become extremely popular consumer electronics devices. Streaming audio to these devices typically requires a device that supports either Bluetooth audio or Apple's AirPlay protocol. Bluetooth audio was selected for this project to ensure that the amplifiers designed for this project supported the widest range of possible audio sources.

There are many different version of Bluetooth and many different Bluetooth profiles. A Bluetooth profile is a specification for a particular feature that can be implemented in Bluetooth devices. For the project described in this paper, a Bluetooth module was utilized that supports Bluetooth v3.0. To stream audio from a Bluetooth audio source to the wireless Bluetooth amplifier, both the audio source and the Bluetooth module on the amplifier must support the Advanced Audio Distribution Profile (A2DP) ${ }^{1}$. For more advanced features, like controlling playback and displaying the artist name and track name, the Bluetooth module must support the Audio/Video Remote Control Profile $(\mathrm{AVRCP})^{2}$.

\section{Project Description}

Each student in the embedded systems course was required to design and implement a complete Bluetooth audio amplifier from schematic capture to PCB assembly. Certain components were pre-selected for the students to ensure interoperability of the components and to reduce the design time to fit into a 12-week semester. Since the pre-selected components were all unfamiliar to the students, the data sheets for these components were provided along with some tips on how to read those data sheets. The importance of carefully and repeatedly reading data sheets was stressed throughout the duration of the project. A high-level diagram of the amplifier project is shown in Figure 1. The requirements for the amplifier and a description of each phase of the project is described in the following sections. 


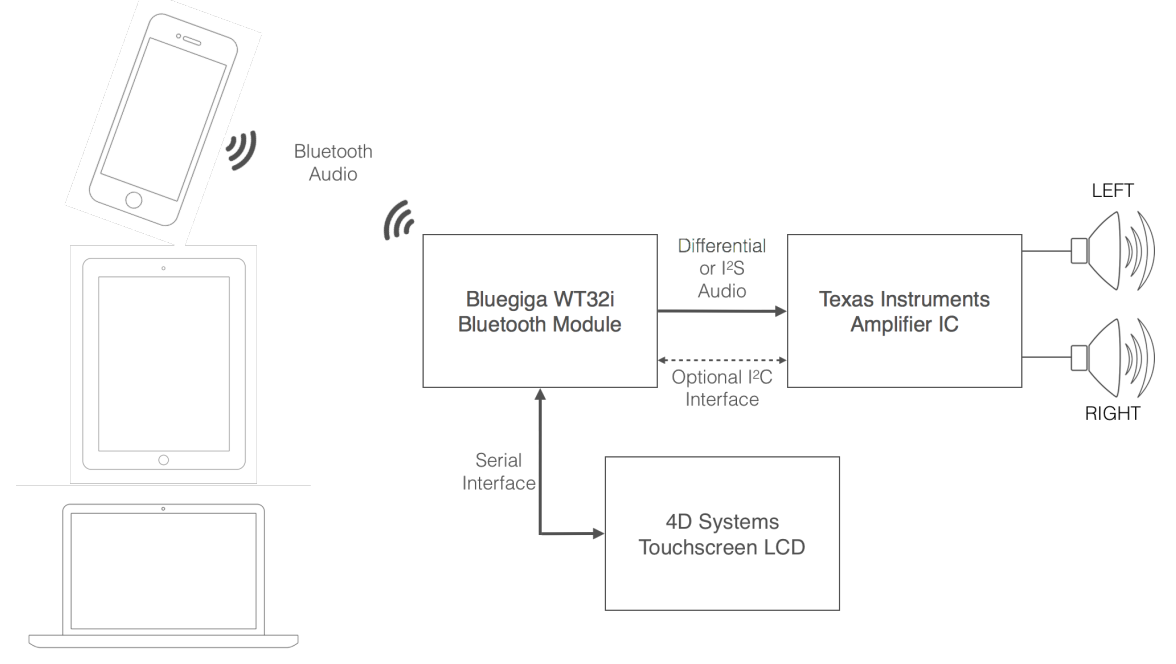

Figure 1: A high-level diagram of the amplifier project

\subsection{Requirements}

All students were required to design a wireless audio amplifier that satisfied the following requirements:

- Utilize a Bluegiga WT32i-A-AI6 Bluetooth Audio Module ${ }^{\frac{3}{3}}$ for Bluetooth connectivity.

- Utilize a Texas Instruments TPA3110D2 15-Watt Stereo Class-D Audio Power Amplifier ${ }^{4}$ to amplify the audio. Optionally, the project may instead utilize a Texas Instruments TAS5766M 20-Watt Class-D Stereo Smart Amp ${ }^{5}$ for a digital audio input and a configurable digital audio processor.

- Utilize a 4D Systems uLCD-43PCT ${ }^{6}$ capacitive touchscreen to display a user interface.

- Display the artist name and track name on the user interface.

- Display the track length and current track position on the user interface.

- Provide playback controls (i.e. play, pause, next track, etc.) on the user interface.

- Provide volume controls (i.e. volume up/down and mute) on the user interface.

- Accept connections from and stream music from any smartphone or device that supports the Bluetooth A2DP profile (includes both Android and iOS devices).

- Power all the components of the amplifier using a single power supply.

While this list of requirements is rather short, selection of the major components narrows the design space sufficiently to ensure that a single instructor can easily manage many different student implementations. 


\subsection{Design and Schematic Capture}

The first phase of the project required students to design and perform schematic capture of their amplifiers. Schematic capture was completed using CadSoft EAGLE PCB Design Software ${ }^{7}$. Students were encouraged to create their schematic in a modular fashion and focus on one major component at a time. For each major component, students referred to the data sheet to create the necessary schematic symbols in EAGLE and select all of the necessary supporting electronic components. Major components were connected with careful consideration for the voltage levels on their communication interfaces. Students were also required to design a power system. Most selected a $12 \mathrm{~V}$ external power source that directly powered the Texas Instruments amplifier IC. The $12 \mathrm{~V}$ source was stepped down to $5 \mathrm{~V}$ using a switching regulator to power the LCD screen. The $5 \mathrm{~V}$ line was subsequently regulated down to $3.3 \mathrm{~V}$ using a linear regulator to power the Bluetooth module. Most student schematics consisted of the following five sections: (1) the Bluetooth module and supporting components, (2) the amplifier IC and supporting components, (3) header pins and supporting components for the external LCD, (4) a buck converter consisting of a switching regulator and supporting components, and (5) a linear regulator and supporting components. A schematic for the amplifier IC portion of the project is shown in Figure 2.

Much of the in-class time for the embedded systems course was treated as lab time during which students could work on their projects and ask questions. However, some class time was spent to introduce students to new concepts and tools and to refresh them on concepts that they had previously learned. During the design and schematic capture phase of the project class time was spent discussing the following topics:

- Creating component symbols and performing schematic capture in CadSoft EAGLE

- Designing a schematic for testing and debug by including debug interfaces, test points, and status LEDs

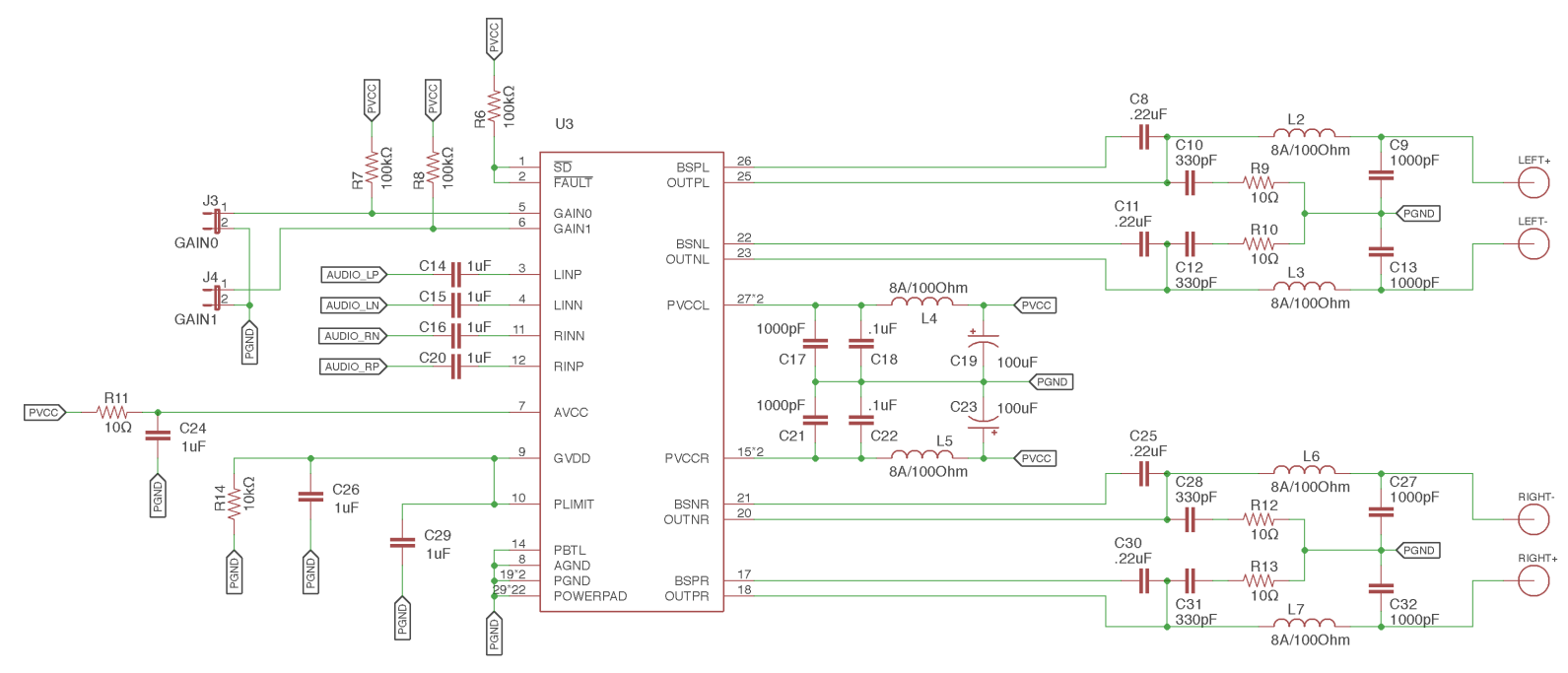

Figure 2: A schematic for the amplifier IC portion of the project 
- Serial, $\mathrm{I}^{2} \mathrm{C}$ (Inter-Integrated Circuit), and $\mathrm{I}^{2} \mathrm{~S}$ (Inter-IC Sound) communication protocols

- Voltage level translators on communication interfaces

- Designing a buck converter using a switching regulator

- Utilizing vendor supported design tools such as Texas Instruments WEBENCH ${ }^{8}$

- Utilizing vendor reference designs

- Project management and maintaining a Bill-Of Materials (BOM)

\subsection{PCB Layout}

The second phase of the project required students to complete a Printed Circuit Board (PCB) layout of their schematic using EAGLE. Prior to starting layout of their PCBs, students created the required land patterns and silk screens (referred to as packages in EAGLE) for any component symbols that they created during the schematic capture portion of the project. All PCB layouts were created as 2-layer boards using either $1 \mathrm{oz}$ or $2 \mathrm{oz}$ copper thickness to ensure that the boards could be manufactured quickly and at low cost. Student PCBs designed to use 1 oz copper were ordered from OSH Park ${ }^{[9}$ while those designed for 2 oz copper were ordered from SeeedStudio ${ }^{10}$. Additionally, solder paste stencils were created and ordered from OSH Stencils ${ }^{11}$ to aid PCB assembly. Figure 3 shows an example of a PCB layout for the amplifier project. Figure 4 shows a topside rendering of the PCB that was generated by OSH Park.

Students taking the embedded systems course had little to no prior experience with PCB layout. During this portion of the project, some class time was used to introduce the students to aspects of PCB layout that related to their projects. A list of the topics covered follows:

- Creating packages and devices that can be used for PCB layout in CadSoft EAGLE

- Component placement in a PCB layout

- Layout considerations for a buck converter

- Layout considerations for Bluetooth antenna and using vias for RF isolation

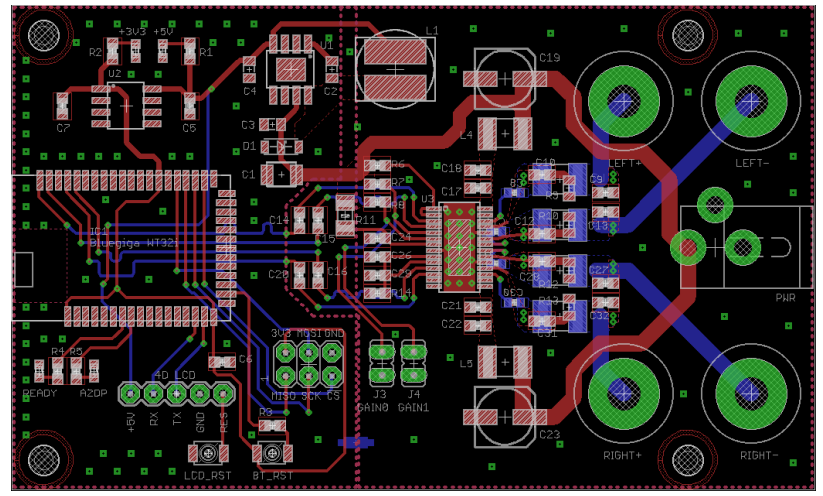

Figure 3: PCB layout for an amplifier project

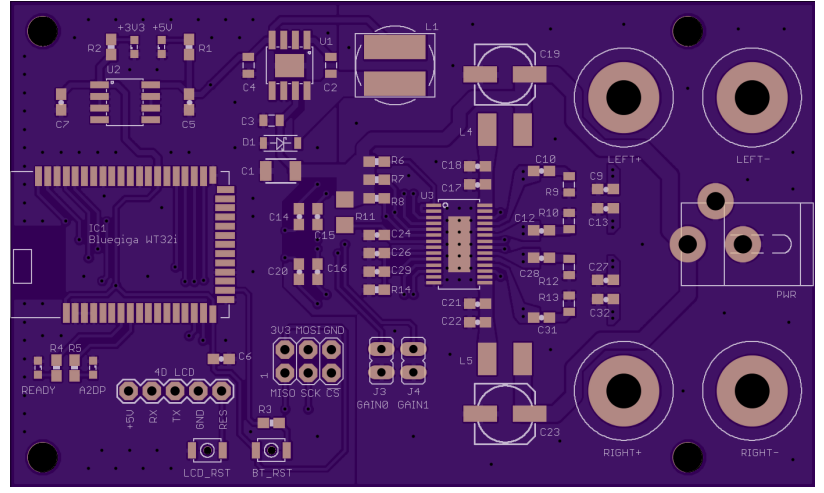

Figure 4: OSH Park rendering of project PCB 
- Utilizing a trace width calculator to determine and set appropriate trace widths

- Creating and connecting multiple ground planes

- Routing power and signals traces, using vias, and differential pair routing

- Utilizing vias to transfer heat between layers of a PCB to improve thermal performance

- Running Design Rule Checks (DRC) and Electrical Rule Checks (ERC)

- Creating Gerber files and ordering PCBs

\subsection{Bluetooth Configuration \& Amplifier Configuration}

While the project PCBs were being manufactured, students switched their focus to the software and configuration portion of the project. The Bluegiga WT32i-A-AI6 Bluetooth Audio Module supports a variety of Bluetooth profiles and features. For this project, students were required to utilize the Bluetooth $\mathrm{A}_{2} \mathrm{DP}^{1}$ and $\mathrm{AVRCP}{ }^{[2}$ profiles. Setting the WT32i module as an A2DP SINK allows the module to receive streaming audio from A2DP sources. Setting the WT32i module as an AVRCP CONTROLLER allows the module to control playback and volume and to get artist name and track name. Other configuration settings supported by the WT32i specify how audio devices connect and disconnect from the module. The module also allows for the selection and prioritization of different audio CODECs including SBC, AAC, and APT-X.

The project requirements gave the students a choice of using either a Texas Instruments TPA3110D2 or a Texas Instruments TAS5766M amplifier IC. The TI TPA3110D2 device is configured using hardware control and is therefore automatically configured when power is applied. However, the more complex TI TAS5766M has configuration registers that must be set by an external source after power is applied. Students that used the TI TAS5766M in their amplifier projects configured the IC by sending commands from the WT32i to the TAS5766M via the optional $\mathrm{I}^{2} \mathrm{C}$ bus shown in Figure 1.

To facilitate this portion of the project, students used a Bluegiga WT32i Hardware Development Kits (part \#DKWT32i). This gave them the ability to familiarize themselves with the available configuration options and test various settings before assembling their own PCBs. Additionally, it also gave the students insight into how hardware development kits can be used to assist in the development process.

During this portion of the project, class time was spent helping students understand the Bluetooth specification and the A2DP and AVRCP profiles. Additional time was spent discussing $\mathrm{I}^{2} \mathrm{C}$ and how to utilize the $\mathrm{I}^{2} \mathrm{C}$ interface on the WT32i to configure external components such as the TAS5766M.

\subsection{Touchscreen LCD User Interface}

Students were required to implement a user interface using a 4D Systems uLCD-43PCT 4.3" LCD capacitive touchscreen. These LCD screens have a programmable 16-bit microprocessor, 
two serial UART ports, an $\mathrm{I}^{2} \mathrm{C}$ interface, 13 general purpose I/O pins, 8 hardware timers, and microSD card support. The screens can be controlled either by programming the built-in microprocessor or by sending serial commands to the device.

For their amplifier projects, students used the 4D Systems Graphics Language (4DGL) $)^{\sqrt{12}}$ to program the LCD screen's built-in microprocessor. Their user interfaces provided artist and track names, media playback controls, and volume control. Interacting with the user interface causes the LCD screen to generate commands that are compatible with the Bluegiga WT32i and then transmit those commands over serial to the Bluetooth module (e.g. AVRCP PLAY and AVRCP MUTE). Bluegiga provides an application note that includes a complete list of supported AVRCP commands $\frac{13}{13}$. In addition to transmitting playback commands, students also programmed their LCD screens to subscribe to AVRCP events such as TRACK CHANGED. These events were used to update the display on the LCD screen. An example user interface with placeholders for track name, artist name, and album name is shown in Figure 5.

The touchscreen LCD screens were designed as an add-on feature for the amplifier projects. That is, students' amplifiers could operate with or without the LCD screen connected. When connected, the LCD screen provided all of the features previously discussed. When the LCD screen was not connected, playback and volume could only be controlled at the audio source. This was used to illustrate how companies can differential product lines and sell devices at different price points.

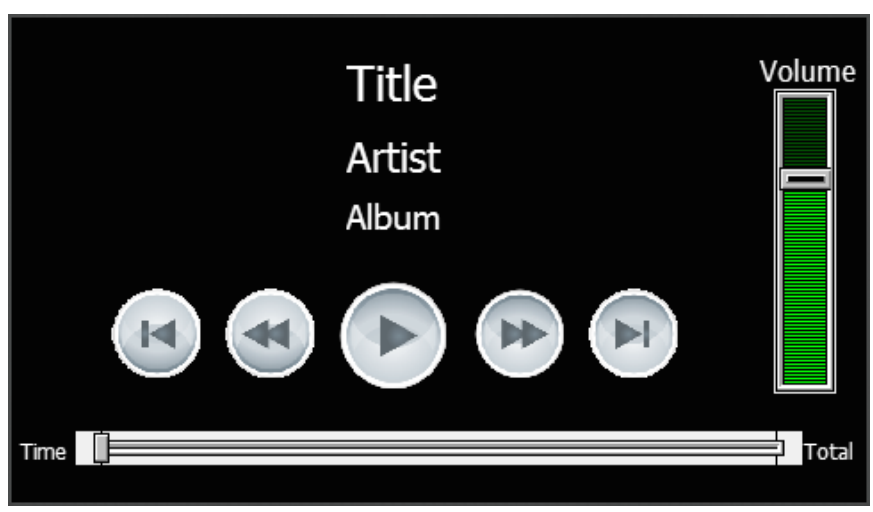

Figure 5: An example user interface

During this portion of the project, class time was spent aiding students in the software development process and in their understanding of 4DGL. Additional time was spent discussing the structure and contents of the AVRCP commands that were transmitted from the LCD screen to the WT32i.

\subsection{PCB Assembly \& Debugging}

The final phase of the project required students to assemble, test, and debug their amplifier projects. Before students' PCBs arrived, each student ordered the components they would need to assemble their board. While all of the students in the course had experience soldering through-hole components, none had experience with surface mount components. For these projects, students were soldering $0.5 \mathrm{~mm}$ or $0.65 \mathrm{~mm}$ pitch amplifier ICs. In some cases, students designed their amplifier projects using components in packages as small as $0402\left(0.04^{\prime \prime} \times 0.02^{\prime \prime}\right)$. However, most students used slightly larger $0603\left(0.06^{\prime \prime} \times 0.03^{\prime \prime}\right)$ and $0805\left(0.08^{\prime \prime} \times 0.05^{\prime \prime}\right)$ components. A variety of different soldering techniques were demonstrated for the students including: 
- hand soldering components and ICs

- drag soldering ICs

- hot air soldering with solder paste

- using stencils, solder paste, and a "reflow oven" (i.e. actually an inexpensive toaster oven)

- desoldering and rework using hot air

- desoldering with low melting point desoldering wire

A microscope with a camera attachment made these demonstrations easily visible to all students in the class. Students were given an opportunity to practice each of these techniques on spare PCBs prior to assembling their own amplifier. Most students opted to take advantage of their stencils and the reflow oven. Figure 6 shows a PCB prior to assembly, while Figure 7 shows an assembled PCB.

During and after assembly of their PCBs, students encountered a wide range of problems that prevented their amplifiers from functioning properly. Some of those problems are listed below:

- components were overheated and damaged during assembly

- land patterns were created incorrectly in EAGLE so components could not be soldered directly to pads

- PCB included extra traces or was missing traces

- incorrect values selected for supporting electronic components

- components were ordered in the wrong physical size (i.e. PCB was designed to use a capacitor in a 1206 package but a capacitor in a 0402 package was ordered)

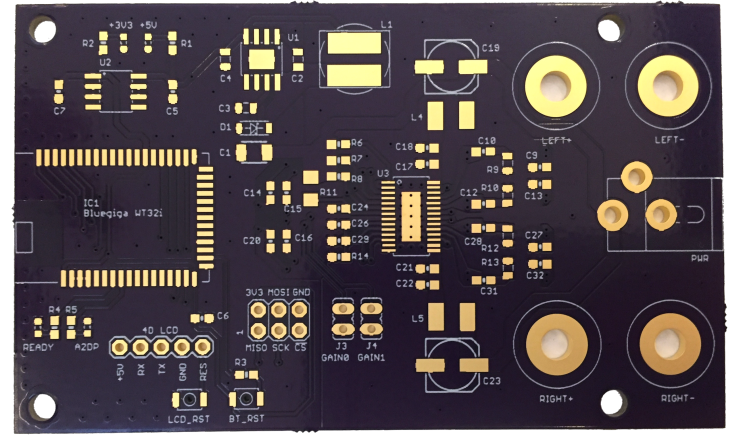

Figure 6: PCB prior to assembling

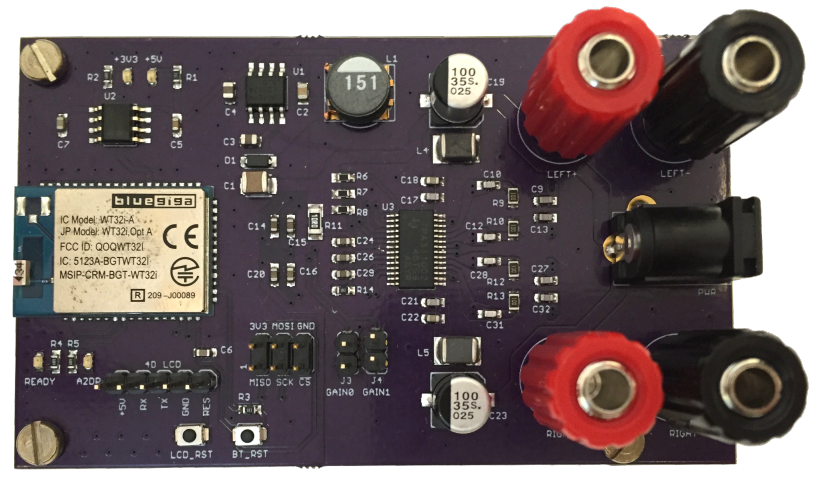

Figure 7: Assembled PCB 


\section{Learning Outcomes}

The amplifier project was used to assess students on a number of ABET learning outcomes. Outcomes were assessed through a combination of faculty observation, submissions of student work, and in-class demos. The assessed learning outcomes and a description of how they apply to the project follows.

- An ability to apply knowledge of mathematics, science, and engineering - Students utilized a wide range of science and engineering skills including circuit design, hardware communication interfaces, software engineering, and programming.

- An ability to design a system, component, or process to meet desired needs within realistic constraints such as economic, environmental, social, political, ethical, health and safety, manufacturability, and sustainability - Students were given an initial set of requirements that constrained their design choices. While the students were not given a specific budget, they were encouraged to think carefully about the cost of their components, their PCBs, and PCB assembly. Using surface mount components, as opposed to through-hole components, reduces the cost of PCB assembly. It also reduces the size of a PCB further reducing the cost to build systems.

- An ability to identify, formulate, and solve engineering problems - Students were given main components but had to design the rest of the amplifier themselves. The whole project is an engineering challenge that requires a range of hardware and software skills to complete.

- A recognition of the need for, and an ability to engage in life-long learning - Throughout the entire project, students were required to continually refer to data sheets and specifications. Reading through data sheets is a skill that the students will carry with them to future projects.

- An ability to use the techniques, skills, and modern engineering tools necessary for engineering practice - Students employed a range of skills including programming and soldering skills. Students utilized a number of different engineering tools for the project including CAD tools, hardware development kits, multimeters, and oscilloscopes.

\section{Discussion}

In hindsight, there are a few changes that would be made were this project attempted again in the future. For the project described in this paper, the students were given due dates for high-level milestones such as completing their schematic capture and completing their PCBs. Each phase of the project had only a single milestone. These high-level milestones made it difficult for the students to stay on schedule, particularly as the deadline for their PCB layouts approached. Finer grained milestones would almost certainly make it easier for students to stay on schedule. That is, instead of having one deadline for schematic capture, there could be separate deadlines for the amplifier IC section of the schematic and another for the Bluetooth module section of the schematic. Instead of having one deadline for completed PCBs, there could be a deadline for 
layout of the amplifier IC and all of its supporting components. There could be another deadline for the layout of the Bluetooth module and another for the power system.

When doing this type of project in a twelve week time frame, it is extremely important that students meet the PCB layout deadline so that the PCBs can be ordered and arrive with sufficient time for students to assemble and debug them. The time from ordering PCBs until they arrive can range from 2 to 4 weeks.

The project, as described, can be modified in a variety of ways. The cost of the project can be reduced by replacing the touchscreen LCD user interface with a less expensive LCD screen that acts as only a display. The touchscreen LCD can also be completely eliminated and replaced with a set of inexpensive momentary switches to provide playback control from the amplifier. A side-effect of eliminating the LCD screen is that it also eliminates the software engineering and programming aspects of the project. Of course, playback control from the amplifier is optional and the feature could be completely eliminated thereby required no LCD screen or momentary switches.

Another modification to the project is to have students work in small groups on specific parts of the project. For example, a group of 2 to 3 students could work on the schematic capture and PCB layout portion of the project. Another small group of students could work on programming the touchscreen LCD user interface. This reduces the total time required for the project. However, it also means that students only get part of the experience of designing a complete embedded system.

\section{Conclusions}

This paper described a Bluetooth audio amplifier project that was completed by undergraduate computer engineering students in an embedded systems course at York College of Pennsylvania. The students had an opportunity to apply knowledge and practice many of the skills that they learned earlier in the engineering curriculum. Additionally, students learned some new skills while developing an embedded system from a list of requirements all the way to PCB assembly. Overall, the students provided positive feedback and found the project to be interesting and engaging.

\section{References}

[1] Bluetooth Special Interest Group. Advanced Audio Distribution Profile Specification, Jul 2012. URL https://www.bluetooth.org/docman/handlers/DownloadDoc.ashx?doc_id=260859 Rev. v13.

[2] Bluetooth Special Interest Group. Advanced Audio/Video Remote Control Profile, Sep 2014. URL 
https://www.bluetooth.org/docman/handlers/DownloadDoc.ashx?doc_id=292286

Rev. v1.6.0.

[3] Bluegiga WT32i Bluetooth Audio Module Product Page, 2015. URL

https://www.bluegiga.com/en-US/products/wt32i-bluetooth-audio-module/. [Last Accessed: Jan 23, 2016].

[4] Texas Instruments. TPA3110D2: 15-W Filter-Free Stereo Class-D Audio Power Amplifier With Speakerguard, 2012. URL http://www.ti.com/lit/ds/symlink/tpa3110d2.pdf. Rev. Jul 2012.

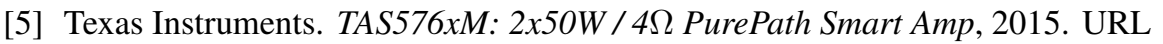
http://www.ti.com/lit/ds/symlink/tas5766m.pdf. Rev. Jan 2015.

[6] 4D Systems. 4.3" microLCD PICASO Display, Aug 2014. URL http: / / www . 4dsystems.com.au/ productpages/uLCD-43/downloads/uLCD-43_datasheet_R_1_5.pdf. Rev. 1.5.

[7] CadSoft EAGLE PCB Design Software, 2015. URL http: / / www • cadsoftusa . com. [Last Accessed: Jan $23,2016]$.

[8] Texas Instruments WEBENCH Design Center, 2015. URL http://www.ti.com/lsds/ti/analog/webench/overview.page. [Last Accessed: Jan 23, 2016].

[9] OSH Park, 2015. URL https: / / oshpark.com. [Last Accessed: Jan 23, 2016].

[10] Seeed Studio, 2015. URL http:// seeedstudio.com. [Last Accessed: Jan 23, 2016].

[11] OSH Stencils, 2015. URL https : / /wWw . oshstencils . com. [Last Accessed: Jan 23, 2016$].$

[12] 4D Systems. 4DGL Programmers Reference Manual, Dec 2013. URL http: / / www . 4 dsystems . com. au/productpages/4DGL/downloads/4DGL_progmanual_R_5_3.pdf. Document Revision: 5.3.

[13] Bluegiga. iWRAP Application Note: Bluetooth A2DP and AVRCP Profiles, Jul 2014. Version 1.7. 